\begin{tabular}{|c|c|}
\hline Title & $\begin{array}{l}\text { Numerical Modeling of Cryogenic Temperature Sensors Based on Plasmonic Oscillations in Metall lic Nanoparticles } \\
\text { Embedded Into Photonic Crystal Fibers }\end{array}$ \\
\hline Author(s) & Florous, Nikolaos John; Saitoh, Kunimasa; Koshiba, Masanori \\
\hline Citation & $\begin{array}{l}\text { IEEE Photonics Technology Letters, 19(5), 324-326 } \\
\text { https://doi.org/10.1109/LPT.2007.891618 }\end{array}$ \\
\hline Issue Date & 2007-03-01 \\
\hline Doc URL & http:/hdl.handle.net/2115/22537 \\
\hline Rights & $\begin{array}{l}\text { (C2007 IEEE. Personal use of this material is permitted. However, permission to reprint/republish this material for } \\
\text { advertising or promotional purposes or for creating new collective works for resale or redistribution to servers or lists, } \\
\text { or to reuse any copyrighted component of this work in other works must be obtained from the IEEE. IEEE, IEEE } \\
\text { PHOTONICS TECHNOLOGY LETTERS, 19, 5, 2007, 324-326 }\end{array}$ \\
\hline Tyре & article \\
\hline File Information & IPTL19-5.pdf \\
\hline
\end{tabular}

Instructions for use 


\title{
Numerical Modeling of Cryogenic Temperature Sensors Based on Plasmonic Oscillations in Metallic Nanoparticles Embedded Into Photonic Crystal Fibers
}

\author{
Nikolaos John Florous, Member, IEEE, Kunimasa Saitoh, Member, IEEE, and Masanori Koshiba, Fellow, IEEE
}

\begin{abstract}
We perform a numerical investigation on the operation of an inline temperature sensor based on the inclusion of metallic nanoparticles into photonic crystal fibers (PCFs). This creates a simple, yet robust, platform which can be used to investigate the properties of nanoparticles, for sensing, spectroscopy, and optical switching applications. The optical response of gold nanoparticles embedded in the PCF matrix was evaluated as a function of temperature and the use of the structure as an inline fiber-optic temperature sensor is theoretically described. A blue-shift in the localized surface plasmon resonance related peak, as well as narrowing of the plasmon resonance, was observed upon decreasing the temperature of the nanoparticle embedded into the PCF at very low temperatures, thus permitting the operation of this sensing platform at cryogenic temperatures.
\end{abstract}

Index Terms-Drude model of metals, liquid sensors, photonic crystal fiber (PCF), sensitivity analysis, temperature-dependent Sellmeier equations, virtual boundary method.

\section{INTRODUCTION}

$\mathbf{O}^{\mathrm{p}}$ PTICAL fibers provide a macroscopic platform for exploiting a wide range of functionalities inherent in nanostructures and nanoparticles. Devices based on optical fibers have several advantages, including immunity to electromagnetic interference, high response times, small size and weight, the ability to be remotely interrogated, and the total absence of free space optics. Development of fiber-optic devices like chemical and biological sensors, based on excitation of surface plasmons in continuous metallic films deposited on the surface of optical fibers, has been extensively reported in the literature [1], [2]. Some recent efforts have also been made to form nanoparticles at the ends of optical fibers to exploit localized surface plasmon (LSP) resonances [3], or have surface enhanced Raman effects [4]. In this study, photonic crystal fiber (PCF)-based optical devices have been rigorously analyzed based on the excitation of localized plasmons in metallic nanoparticles, incorporated inside the PCF matrix. Our investigation differs significantly from those based on the excitation of surface plasmons in continuous metallic films deposited on the surface of a conventional fiber, or at the end of the fiber. A clear improvement of employing metallic nanoparticles instead of the continuous thin films studied in attenuated total

Manuscript received September 15, 2006; revised December 26, 2006. This work was supported by the Japan Society for the Promotion of Science (JSPS).

The authors are with the Division of Media and Network Technologies, Hokkaido University, Sapporo 060-0814, Japan (e-mail: nflorous@dpo7.ice. eng.hokudai.ac.jp; ksaitoh@ist.hokudai.ac.jp; koshiba@ist.hokudai.ac.jp).

Color versions of one or more of the figures in this letter are available online at http://ieeexplore.ieee.org.

Digital Object Identifier 10.1109/LPT.2007.891618 reflection sensors is that the shape as well as the polarization requirements can be significantly relaxed. Moreover, unlike surface modes in surface plasmon resonance which cannot be excited by propagating light due to a phase mismatch between the propagating light and surface plasmon waves, LSPs can be excited by propagating light. On the other hand, the use of PCFs increases the possibility of engineering their modal properties [5], and significantly enhances its thermal response (depending on the material inserted into the air-holes) in comparison to conventional fibers [6]. In this theoretical study, ellipsoidal gold nanoparticles $(\mathrm{Au})$ incorporated inside a PCF matrix enable the formation of cryogenic temperature sensors, the effective characterization of several novel material platforms, and the potential formation of optical switches. We rigorously characterize the collective oscillations of metallic particles embedded into a PCF and we investigate the corresponding temperature sensing in a detailed manner. As metallic nanoparticles are heated or cooled, the position of the plasmon resonance peak shifts and the peak broadens. Thus, by incorporating gold nanoparticles within the core of a PCF, these phenomena form the basis of an in-line PCF temperature sensor.

\section{Modeling the Thermooptical Response of MEtalliC NANOPARTICLES EMBEDDED INTO A PCF MATRIX}

Resonant light scattering and absorption by metallo-dielectric nanoparticles can be well estimated by the Mie theory. The plasmon resonance wavelength, in principle, depends on the size, shape, and dielectric constant of the nanoparticles and on the dielectric constant of the medium surrounding the nanoparticles. As these refractive indexes and localized plasmon oscillations are dependent on temperature, the development of a fiber-optic temperature sensor that is based on sensing the shift in the plasmon resonance wavelength with temperature is proposed. In order to rigorously investigate the temperature dependence of resonances associated with LSPs, analytical calculations for evaluating the extinction cross section $C_{\text {ext }}$ of gold nanoparticles embedded in a PCF-matrix were carried out. The effects of nanoparticle size and shape on LSP-related resonances were also taken into account in our calculations. In the analytical calculations presented below, the size of the gold nanoparticles was varied from 30 to $50 \mathrm{~nm}$ and the volume fraction $f$ of the particles in the medium was taken to be very small, i.e., $f \ll 1$. Consider the PCF structure as shown in Fig. 1. For simplicity, we consider a PCF with two air-hole rings, having pitch constant $\Lambda=35 \mu \mathrm{m}$, and air-hole diameter $d=14 \mu \mathrm{m}$, for preserving its single-mode operation. Based on the quasistatic theory, in the regime where $f \cong 0.01 \%$, assuming a Gaussian-like pulse propagating along the PCF and interacting 


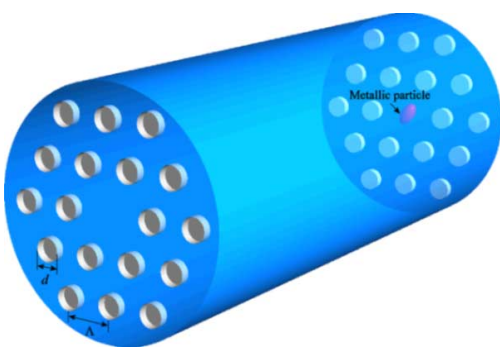

Fig. 1. Schematic diagram of the PCF considered as an inline cryogenic temperature sensor with a metallic nanoparticle embedded into the fiber.

with the nanoparticle, the extinction ratio under such a scenario is particle-geometry-dependent and is defined for an ellipsoidal nanoparticle as [7]

$$
\begin{aligned}
C_{\mathrm{ext}}= & \frac{24 \pi^{2} \alpha_{1} \alpha_{2} \alpha_{3}}{\lambda} \\
& \times \frac{\varepsilon_{2}^{3 / 2} \varepsilon_{m b}}{\left(3 \varepsilon_{2}-3 \varepsilon_{2} L_{i}+3 \varepsilon_{m a} L_{i}\right)^{2}+9 L_{i}^{2} \varepsilon_{m b}^{2}} \\
& +\frac{128 \pi^{5} \varepsilon_{2}^{2} \alpha_{1}^{2} \alpha_{2}^{2} \alpha_{3}^{2}}{3 \lambda^{4}} \\
& \times \frac{\left(\varepsilon_{m a}-\varepsilon_{2}\right)^{2}+\varepsilon_{m b}^{2}}{\left(3 \varepsilon_{2}-3 \varepsilon_{2} L_{i}+3 \varepsilon_{m a} L_{i}\right)^{2}+9 L_{i}^{2} \varepsilon_{m b}^{2}} \\
L_{i}= & \frac{\alpha_{1} \alpha_{2} \alpha_{3}}{2} \\
& \times \int_{0}^{+\infty} \frac{d s}{\left(s+\alpha_{i}^{2}\right) \sqrt{\left(s+\alpha_{1}^{2}\right)\left(s+\alpha_{2}^{2}\right)\left(s+\alpha_{3}^{2}\right)}}
\end{aligned}
$$

where $\varepsilon_{m}=\varepsilon_{m a}+j \varepsilon_{m b}$ is the electric permittivity of the metallic nanoparticle, $\varepsilon_{2}$ is the effective electric permittivity of the PCF, and $L_{i}$ is a depolarization factor depending on the orientation of the incident field with respect to the $i$ th axis of the ellipsoid nanoparticle $(i=1,2,3)$. In the context of ellipsoidal metallic nanoparticles deposited inside PCFs, the fiber axis was assumed to be in the direction of the semiaxis $\alpha_{3}$ of the ellipsoidal metallic nanoparticle. Moreover, in the theoretical calculations described above, the incident field was taken to be parallel to the semiaxis $\alpha_{1}$. The electric permittivity of the metallic (gold, Au) nanoparticle is characterized by a Drude-Sommerfeld model of frequency and temperature dependence [8]

$$
\begin{aligned}
\varepsilon_{m} & (\omega, T) \\
\quad= & \varepsilon_{m a}(\omega, T)+j \varepsilon_{m b}(\omega, T) \\
= & 1-\varepsilon_{\infty} \frac{\omega_{p}^{2}(T)}{\omega^{2}+\omega_{c}^{2}}+j \varepsilon_{\infty} \frac{\omega_{p}^{2}(T) \omega_{c}(T)}{\omega\left\{\omega^{2}+\omega_{c}^{2}(T)\right\}} \\
\omega_{p}(T) & \\
= & {\left[\frac{e^{2}\left\{\frac{N\left(T_{0}\right)}{1+3 \gamma\left(T-T_{0}\right)}\right\}}{m * \varepsilon_{0}}\right]^{1 / 2} } \\
\omega_{c}(T) & \\
= & \frac{A V_{f}}{\max \left\{a_{i}\right\}}+\frac{\omega_{p}^{2} \varepsilon_{0}}{\sigma\left(T_{\theta}\right)} \frac{\left[\frac{1}{10}+\left(\frac{T}{T_{\theta}}\right)^{5} \int_{0}^{T / T_{\theta}} \frac{x^{4}}{e^{x}-1} d x\right]}{\int_{0}^{1} \frac{x^{5}}{\left(e^{x}-1\right)\left(1-e^{-x}\right)} d x} \\
& +\frac{1}{12} \pi^{3} \frac{\Gamma M}{\hbar E_{f}}\left[\left(k_{B} T\right)^{2}+\left(\frac{\hbar \omega}{2 \pi}\right)^{2}\right]
\end{aligned}
$$

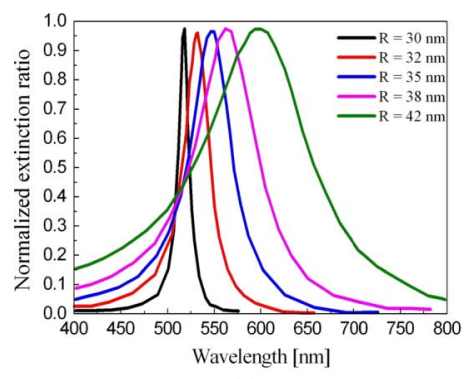

(a)

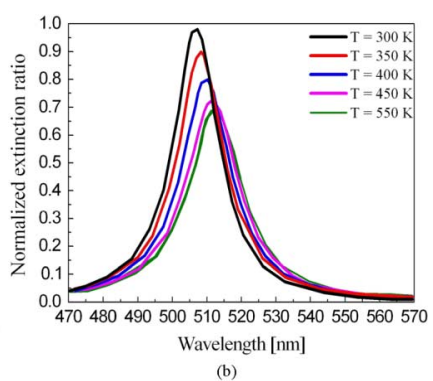

Fig. 2. (a) Normalized extinction ratio as a function of the operational wavelength $\lambda$ in nanometers, for constant axis $\alpha_{2}=\alpha_{3}=34 \mathrm{~nm}$ and variable axis $\alpha_{1}=R$, at room temperature of $T=300 \mathrm{~K}$; (b) normalized extinction ratio as a function of the wavelength for spherical nanoparticles of radius $R=34 \mathrm{~nm}$ at different temperatures $T$.

where $N\left(T_{0}\right)=5.9 \times 10^{22} \mathrm{~cm}^{-3}$ stands for the density of electrons at a reference temperature of $T_{0}=300 \mathrm{~K}, \omega$ being the angular frequency, $m^{*}$ stands for the electron's effective mass, $\gamma$ is the linear expansion coefficient of metals, $\varepsilon_{\infty} \cong 1$ is the dielectric constant of the metal at sufficient high frequencies, $\omega_{p}$ is the plasma frequency, $\omega_{c}$ is the electron collision frequency in the free electron model of metals, $T$ is the absolute temperature in Kelvin, $k_{B}$ is the Boltzman's constant, $\hbar$ is the normalized Plank's constant, $E_{f}$ is the electron Fermi energy ( $E_{f}=5.51 \mathrm{eV}$ for gold- $\mathrm{Au}$ ), $T_{\theta}=185 \mathrm{~K}$ is the Debye temperature for gold, $\sigma\left(T_{\theta}\right)=1.3 \times 10^{-8} \Omega \mathrm{m}$ is the dc conductivity of gold defined at the Debye temperature, $\Gamma=0.55$ stands for the scattering probability over the Fermi surface, $M=0.78$ represents the fractional umklapp scattering, $V_{f}=1.39 \times 10^{6} \mathrm{~ms}^{-1}$ is the Fermi velocity for gold, $\max \left\{\alpha_{i}\right\}$ stands for the maximum semiaxis of the nano-particle, and $A$ is a constant associated with the size effect of the nanoparticles, where for spherical particles $A=1$, while for ellipsoidal particles $A=0.69$. Notice that this model depends also on the size characteristics of the nano-particle considered here, thus, the most accurate one, and, to the best of our knowledge, will be used for the first time for thermoplasmonic studies in PCF-based sensors. The PCF's core is made up from silica with electric permittivity depending on frequency and temperature as described in [6].

\section{Selected Numerical Results}

Fig. 2(a) shows the normalized extinction ratio as a function of the operational wavelength $\lambda$ in nanometers, for dry air as a material inside the air-holes of the PCF, where the effective electric permittivity $\varepsilon_{2}$ was obtained by an accurate modal analysis of the PCF based on the virtual boundary method [9], taking into account the material dispersion effects, while we have considered the following geometrical characteristics of the nanoparticle: $\alpha_{2}=\alpha_{3}=34 \mathrm{~nm}$ and variable axis $\alpha_{1}=R$, at a room temperature of $T=300 \mathrm{~K}$. We can clearly observe that as soon as the major axis of the nanoparticle increases the plasmon resonance is red-shifted. Fig. 2(b) shows the normalized extinction ratio as a function of the wavelength for spherical nanoparticles of radius $\alpha_{1}=\alpha_{2}=\alpha_{3}=R=31 \mathrm{~nm}$ at different temperatures $T$. One can observe that the plasmon resonance related peak shifts to higher wavelengths as the temperature is increased. Moreover, the width of the plasmon resonance related peak also increases upon increasing the temperature and this could be attributed to the strong temperature dependence of 


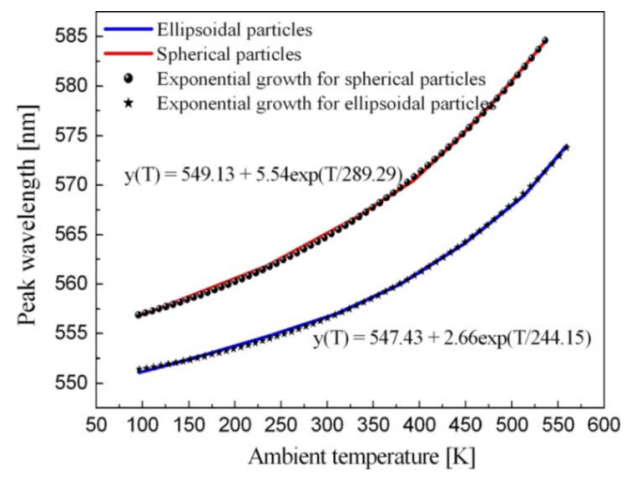

Fig. 3. Impact of the ambient temperature in Kelvin to the peak wavelength shift in nanometers. The sensitivity dependence on the ambient temperature follows an exponential growth with detailed functional characteristics inside the graph.

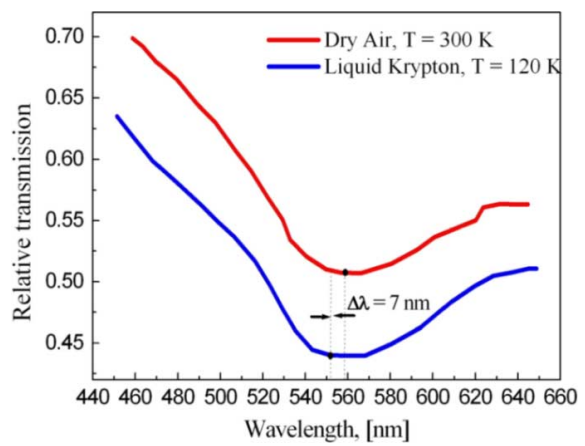

Fig. 4. Impact of the cryogenic cooling to the relative transmission of the plasmonic shift using liquid $\mathrm{Kr}$ (blue lower curve). Observe the blue-shift corresponding to the plasmonic mode in comparison to dry air (red upper curve).

the electron collision frequency $\omega_{c}$. Fig. 3 shows the effect of temperature on the LSP resonance peaks. This dependence can be well described by exponential growths (circles and stars) to the numerical data (blue lower curve and red upper curve).

The ellipsoidal nanoparticle was found to be a bit less sensitive to the thermal fluctuations than the spherical particle. A fair estimation regarding the temperature accuracy is definitely associated with the corresponding accuracy of the temperature-dependent permittivities for metals and for silica we are using in our model. This practically means a temperature range from $100 \mathrm{~K}$ up to $500 \mathrm{~K}$. To study the transmission shift, we next consider the holes of the proposed PCF to be filled with liquid krypton $(\mathrm{Kr})$ with detailed thermooptical material dispersion depicted from [10]. The relative transmission of light in the PCF, taking also into account the scattering as well as the material losses of the nanoparticle, is shown in Fig. 4, for spherical nano-particles of radius $R=38 \mathrm{~nm}$, and for the reference room temperature corresponding to dry air (red upper curve), and to the cryogenic temperature corresponding to liquid $\mathrm{Kr}$ (blue lower curve). The total length of the PCF was taken to be $3 \mathrm{~cm}$ while the total attenuations deduced solely by the fiber structure are $0.00504 \mathrm{~dB}$ at wavelength of $\lambda=550 \mathrm{~nm}$ for dry air and $0.008 \mathrm{~dB}$ for liquid Kr. Fig. 4 shows the exact effect of cooling the PCF using liquid $\mathrm{Kr}$. We can clearly see that there is a blue-shift of about $\Delta \lambda=7 \mathrm{~nm}$ of the corresponding

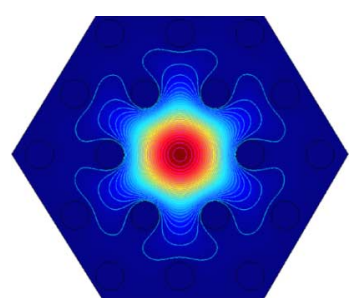

Fig. 5. Normalized electric field distribution at the plasmonic peak wavelength of $\lambda=552 \mathrm{~nm}$, at cryogenic temperature of $T=120 \mathrm{~K}$.

plasmonic resonance in the transmission spectra, as soon as the temperature decreases to cryogenic levels. This phenomenon is essential and suggests a novel application regime of the prescribed PCF platform as an inline cryogenic temperature sensor. The confinement of light in the core of the PCF, at the operational wavelength of $\lambda=552 \mathrm{~nm}$, where the plasmonic mode for liquid $\mathrm{Kr}$ occurs, is shown in Fig. 5, by plotting the normalized electric field distribution. We can clearly observe that the light is well confined even at ultralow temperatures.

\section{CONCLUSION}

A rigorous study on the optical properties of metallic nanoparticles embedded into the core of a PCF has shown that a new technological regime of PCFs could be developed, namely to make a PCF-based temperature sensor that could be used for remote monitoring of temperatures in different media. The obtained resolution of the LSP peak is about $0.062 \mathrm{~nm} / \mathrm{K}$ for the case of spherical nano-particles, and $0.046 \mathrm{~nm} / \mathrm{K}$ for the case of ellipsoidal nano-particles, results which are indicating a fairly fine resolution. The feasibility of the proposed sensor may rely on the electron-beam evaporation process for accurate deposition of the gold nano-particles into the core of the PCF. The proposed scheme may be useful in sensor systems such as photonic Bragg gratings, where it would be advantageous to have a sensor that can operate at cryogenic temperature levels.

\section{REFERENCES}

[1] A. J. Haes Van Duyne, "Plasmonics: A route to nanoscale optical devices," Anal. Bioanal. Chem., vol. 379, pp. 920-930, Aug. 2004.

[2] J. Homola, S. S. Yee, and G. Gauglitz, "Surface plasmon resonance sensors: Review," Sens. Actuators B, vol. 54, pp. 3-15, Jan. 1999.

[3] K. Mitsui, Y. Handa, and K. Kajikawa, "Optical fiber affinity biosensor based on localized surface plasmon resonanse," Appl. Phys. Lett., vol. 85, pp. 4231-4233, Nov. 2004.

[4] D. J. White and P. R. Stoddart, "Nanostructured optical fiber with surface-enhanced Raman scattering functionality," Opt. Lett., vol. 30, pp. 598-600, Mar. 2005.

[5] J. C. Knight, "Photonic crystal fibers," Nature, vol. 424, pp. 847-851, Aug. 2003.

[6] N. J. Florous, S. K. Varsheney, K. Saitoh, and M. Koshiba, "Thermooptical sensitivity analysis of highly birefringent polarimetric sensing photonic crystal fibers with elliptically elongated veins," IEEE Photon. Technol. Lett., vol. 18, no. 15, pp. 1663-1665, Aug. 1, 2006.

[7] H. C. Van de Hulst, Light Scattering by Small Particles. New York: Wiley, 1957.

[8] T. Okamoto, Near-Field Optics and Surface Plasmon Polaritons. Berlin, Germany: Springer, 2001.

[9] N. Florous and M. Koshiba, "Virtual boundary method for the analysis of elliptically cross-sectional photonic crystal fibers with elliptical pores," J. Lightw. Technol., vol. 23, no. 4, pp. 1763-1773, Apr. 2005.

[10] A. C. Sinnock and B. L. Smith, "Refractive indexes of the condensed inert gases," Phys. Rev., vol. 181, pp. 1297-1307, May 1969. 